\title{
Transcranial magnetic stimulation and movement of aneurysm clips
}

Keywords:

TMS

Aneurysm clip

Major depressive disorder

\section{Transcranial magnetic stimulation and movement of aneurysm clips}

We are interested in whether transcranial magnetic stimulation (TMS) could cause movement of intracranial aneurysm clips. We conducted some backyard exercises - albeit, with the encouragement of Prof Anthony Barker, who designed the first TMS machine.

Scrounging through a neurosurgeon's glove box and the first author's garden shed, we collected a titanium aneurysm clip (Yasargil, standard; $2.65 \mathrm{~mm}$ long) and various pieces of wire and safety pins. We bent and trimmed the wire and pins into approximations of the clip. Using weak and strong hand magnets, we selected 6 objects, two being strongly magnetic, two being weakly magnetic and two (one of which was the clip) being non-magnetic (as far as could be detected, they were indifferent to the strong hand magnet). We then conducted some basic investigations, which were filmed and are available as supplementary data.

\section{Ballistic pendulum exercise}

The ballistic pendulum has been used previously in such studies [1]. We hung the weakly magnetic objects from the ceiling on 1.5 meters of sewing cotton (about $1.5 \mathrm{~cm}$ apart). A MagPro (MagVenture) figure- 8 coil was placed approximately $1 \mathrm{~cm}$ away. When single pulses (100\% machine output) were fired, these objects were repelled $0.5-3.5 \mathrm{~cm}$; when a $10 \mathrm{~Hz}$ train was fired, they jumped further away, in an erratic manner. When the highly magnetic objects, were subjected to single and trains of magnetic pulses, they were repelled up to $25 \mathrm{~cm}$.

However, when the non-magnetic objects were exposed to the same conditions (as far as could be detected by the naked eye) they did not move. At times these objects revolved slightly, but we interpreted this to be caused by air movement in an airconditioned room.

\section{Cantaloupe exercise}

We cut a cantaloupe (muskmelon, rock melon) in half and removed the seeds. We pushed the aneurysm clip into the flesh, at right angles to the surface of the flesh. It proceeded until contacting the inner surface of the skin, and resisted further penetration. The pushed end of the clip could be seen protruding slightly above the flesh. In the same manner and about $1.5 \mathrm{~cm}$ away, we pushed a highly magnetic comparator into the fruit.

We placed the face of the coil against the outer surface of the skin, and administered, at 100\% machine output, 75 trains of $4 \mathrm{sec}-$ onds and $10 \mathrm{~Hz}$. After 3000 pulses (to the naked eye) neither the titanium clip nor the highly magnetic comparator had moved.

We had not expected the titanium clip to move, but we had thought the highly magnetic comparator might emerge from the flesh. It did not.

The cantaloupe may have been pre-ripe and gripped these objects too firmly. However, it had been purchased at a retail outlet and was soft and juicy in the mouth.

\section{Egg exercise}

The brain has been described as having "the consistency of a cold boiled egg" [2]. Due to the uncertain grip strength of cantaloupe, we tested the effect of TMS on objects inserted into a 3-cm slab of cold boiled egg.

We boiled a $600 \mathrm{gm}$ free range egg for 4 minutes, removed one side of the shell and cut away soft tissue to produce the desired dimension. We inserted the clip and the highly magnetic comparator into the soft tissue, such that they touched the inside of the shell at roughly $90^{\circ}$, and their outer ends were just visible. We placed the coil against the outside of the shell and administered 75 trains at $100 \%$ of machine output.

This exercise confirmed an earlier exercise, neither clip moved, as far as we could determine with the naked eye (the first author was wearing visual aids, as required for driving).

\section{Discussion}

We could detect no movement of a clip, suspended as a $1.5 \mathrm{~m}$ pendulum, in response to single pules or trains of TMS. Nor could we detect any movement of a clip and a non-magnetic comparator, when they were inserted into the flesh of a cantaloupe, or a boiled egg, and subjected to 3000 pules at $100 \%$ machine output.

Two features of this study provide margins for error. First, in all cases, the face of the coil was never more than $1 \mathrm{~cm}$ away from the clip - in life, this distance would always be more than $3 \mathrm{~cm}$. Second, in this study we used $100 \%$ of machine output - in life, the use of greater than $80 \%$ is almost unknown.

While this study was of the backyard variety, it nevertheless raises the question of whether we worry a little too much about the possibility of TMS induced movement of non-magnetic intracranial aneurysm clips. 
This study was made into a movie: https://www.youtube.com/ watch? $\mathrm{v}=\mathrm{nZrSOXeg} 3 \mathrm{JQ}$.

\section{Conflict of interest}

There are no conflicts of interest.

\section{Appendix A. Supplementary data}

Supplementary data related to this article can be found at http:// dx.doi.org/10.1016/j.brs.2017.08.009.

\section{References}

[1] Barker A. An introduction to the basic principles of magnetic nerve stimulation. J Clin Neurophysiol 1991;8:26-37.
[2] Pridmore S. Download of psychiatry. 2015 [Chapter 2]: page 1, http://eprints. utas.edu.au/287/. [Accessed 29 August 2017].

Saxby Pridmore Discipline of Psychiatry, University of Tasmania, Hobart, Tasmania, Australia

Fiona Lawson

TMS Department, Saint Helen's Private Hospital, Hobart, Tasmania, Australia

* Corresponding author. E-mail address: s.pridmore@utas.edu.au (S. Pridmore).

10 August 2017 Available online $\mathrm{xxx}$ 